\title{
Estudio de la exclusión educativa y abandono en la enseñanza secundaria en algunas instituciones públicas de Costa Rica
}

\author{
Education Exclusion and Dropping out in Public Schools of Costa Rica
}

\author{
Wendy Jiménez Asenjo ${ }^{1}$ \\ Departamento de Estudios e Investigación Educativa \\ Ministerio de Educación Pública \\ San José, Costa Rica \\ wendyjimenezasenjo@gmail.com \\ Marcelo Gaete Astica \\ Departamento de Estudios e Investigación Educativa \\ Ministerio de Educación Pública \\ San José, Costa Rica \\ marcelo.gaete.astica@mep.go.cr
}

Recibido 24 de setiembre de 2012 • Corregido 17 de febrero de 2013 • Aceptado 13 de marzo de 2013

Resumen. Este es un artículo que informa de los hallazgos de la investigación Exclusión educativa y abandono (deserción) escolar en la enseñanza secundaria en Costa Rica, efectuada por Wendy Jiménez y Marcelo Gaete, funcionarios del Departamento de Estudios e Investigación Educativa del Ministerio de Educación Pública, en torno a los factores de exclusión y deserción escolar en el Tercer Ciclo y en el Ciclo de Enseñanza Diversificada de Costa Rica, durante el año 2009. El estudio se realizó por medio de un trabajo de campo realizado en 22 instituciones de secundaria de las diferentes regiones educativas del país. En cada una de las instituciones visitadas se entrevistó, con base en la respectiva guía, al director y al orientador, y se realizaron grupos focales con estudiantes, y padres y madres de familia, con la finalidad de comprender el significado, tal cual el naturalismo y el enfoque cualitativo sugiere, que los distintos de actores de la comunidad educativa -directores, orientadores, estudiantes y padres y madres de familia- dan al proceso de exclusión y deserción educativa. Un aspecto conceptual importante que examina este trabajo es la problematización del concepto de deserción y exclusión, generalmente, de forma acrítica para caracterizar este fenómeno.

Palabras claves. Exclusión educativa, abandono escolar, retención escolar, deserción, Revista Electrónica Educare, Costa Rica.

1 Egresada de la Maestría en Educación para Sexualidad Humana del Centro para la Atención de la Salud de la Sexualidad Humana (CEAS) de Guadalajara, México. Licenciada en Sociología de la Universidad de Costa Rica. Investigadora del Departamento de Estudios e Investigación Educativa, Ministerio de Educación Pública. Posee artículos publicados en las áreas de educación.

2 Máster en Antropología de la Universidad de Costa Rica. Licenciatura en Antropología de la Universidad de Costa Rica. Investigador del Departamento de Estudios e Investigación Educativa, Ministerio de Educación Pública. Ha laborado como como profesor universitario en la carrera de Antropología de la Universidad de Costa Rica, Sede Rodrigo Facio y sede Turrialba. Experiencia en asesorías de impacto ambiental, evaluación de proyectos de desarrollo e investigación social y educativa. Posee artículos publicados en las áreas de educación. 
URL: http://www.una.ac.cr/educare

CORREO: educare@una.cr

\begin{abstract}
This paper reports on the findings obtained from a study conducted in 2009: "Exclusión educativa y abandono (deserción) escolar en la enseñanza secundaria en Costa Rica" [Education Exclusion and Dropping out in Costa Rica], focused on the students from the $7^{\text {th }}$ to the $11^{\text {th }}$ grades of the General Basic Education. For this research, fieldwork was carried out in 22 institutions, in different educational regions of the country. Interviews were conducted with the school principals and counselors in each of the institutions visited. Focal groups were organized with students and parents in order to -as naturalism and qualitative focus suggest -understand the meaning of education exclusion and dropping out from the point of view of the different agents involvedprincipals, counselors, students, parents. A relevant conceptual aspect included in this study is the analysis of the problems related to dropping out and exclusion, generally, in a nonjudgmental way, to characterize this phenomenon.
\end{abstract}

Keywords. School exclusion, dropping out, school retention rate, Educare Electronic Journal, Costa Rica.

\title{
Introducción
}

La problemática de la exclusión educativa o abandono escolar del sistema educativo costarricense es motivo de preocupación nacional y, en particular, para el Ministerio de Educación Pública (MEP), por sus efectos en la configuración de situaciones de pobreza, estado que, sin educación, es difícil superar. Por esa razón, el Departamento de Estudios e Investigación del Ministerio de Educación Pública estimó necesario desarrollar un estudio que contribuya en la comprensión y explicación cualitativa de esta problemática, y que aporte insumos efectivos para la toma de decisiones políticas orientadas a su reducción significativa.

El siguiente artículo informa de los hallazgos alcanzados por medio de la investigación Exclusión educativa y abandono (deserción) escolar en la enseñanza secundaria en Costa Rica en torno al fenómeno de la exclusión/deserción escolar en la enseñanza media, realizada, entre el año 2009 y 2010, con estudiantes, docentes, directores y padres de familias de 22 instituciones de secundaria, de todas las regiones educativas del país que en ese momento existían.

En este artículo se examinan los factores que permiten la comprensión y explicación de este pernicioso fenómeno educativo, poniéndose especial cuidado en observar la gestión institucional, el rol de la familia, y la visión que tienen los estudiantes y las estudiantes. La metodología de investigación seguida se ha basado en el enfoque cualitativo naturalista de investigación.

El problema de la deserción escolar -que en este artículo preferimos denominar como exclusión educativa y abandono escolar o inasistencia, dada la carga semántica que la palabra deserción tiene- no es, en modo alguno, un fenómeno nuevo ni propio de Costa Rica. En el año 2008 este fenómeno alcanzo la cifra del 12\% entre los centros académicos diurnos, porcentaje alrededor del cual giró entre los años 2001 y 2008 (MEP-Departamento de Análisis Estadístico, 2009).

La ausencia a las instituciones educativas, la inasistencia a clases y el abandono escolar por parte de niños, niñas y jóvenes es un problema que afecta a un número relativamente 
importante de jóvenes. Los estudiantes y las estudiantes que no asisten regularmente a las instituciones escolares, lo que debieran hacer por su edad, representan un fenómeno que tiene múltiples causas. No se hace referencia, aquí, a los jóvenes y las jóvenes que no están matriculados, ni entraron a una institución educativa, que son a los que la cobertura no llega, sino a los que ingresaron y se fueron o abandonaron la institución.

Este fenómeno educativo tiene -sin lugar a dudas- diversas causas y factores que lo explican. Entre otras, se señalan las de origen económico (el trabajo o la búsqueda de trabajo para ayudar en el hogar), la ausencia de establecimientos educativos cercanos (genera deslocalización a algunos jóvenes), los problemas familiares (realización de quehaceres en el hogar, embarazo, maternidad, y se puede agregar violencia intrafamiliar y drogas), falta de interés del estudiante y de los padres de familia por el estudio y los problemas de desempeño escolar (Programa de promoción de la reforma educativa en América Latina y el Caribe [PREAL], 2003). Su abordaje requiere, entonces, de aproximaciones parciales, de elecciones temáticas y metodológicas, y obliga a concentrarse en unos aspectos y a marginar otros, para producir un conocimiento idóneo: explicativo o comprensivo. Tal es el caso de la opción de estudiar la deserción ligada al rol de la familia, a la gestión institucional y a los condicionamientos sociales y económicos de los procesos escolares.

Este estudio tuvo como objeto de interés la comprensión factorial del fenómeno de la exclusión-deserción/abandono educativo, en particular el que se presenta en el III Ciclo de la Enseñanza General Básica y Enseñanza Diversificada.

Para alcanzar este objetivo, se indaga en aspectos como la estadística de la exclusión/ deserción, la fases del proceso de exclusión/deserción, la identificación de las razones de este proceder a escala individual, la caracterización del tipo de apoyo que brinda el sistema educativo, el rol de la familia, entre otros.

Finalmente, y luego de la sistematización pertinente, se presentan las conclusiones respectivas y algunas recomendaciones surgidas de la voz de los actores de la comunidad educativa.

\section{Elementos conceptuales de la problemática del abandono/deserción escolar}

La problemática de la "deserción" estudiantil en la enseñanza secundaria no se presenta a la mirada pública como un fenómeno de fácil comprensión, y ello es así porque el lenguaje impide a veces su claro conocimiento. Es necesario, entonces, realizar una reflexión sobre la terminología que se usa para explicar este fenómeno, la que nos ayudará a comprender ya no solo las confusiones terminológicas, sino más profundamente la realidad de este fenómeno, el cual, a veces, el lenguaje no transparenta adecuadamente.

El abandono escolar o la deserción son dos conceptos que se han utilizado para referirse a un fenómeno de la educación secundaria que tiene que ver con el hecho de que un gran 
URL: http://www.una.ac.cr/educare

CORREO: educare@una.cr

número de jóvenes se retiran de las aulas y del sistema educativo. El abandono escolar es un fenómeno en el que concurren muchos factores, unos considerados condicionantes del fenómeno y otros estructurales, determinantes.

En cuanto los factores condicionantes, se entiende por condición una "situación o circunstancia indispensable para la existencia de otra" (Diccionario de la Real Academia Española [DRAE], 2001), es decir, se trata de una situación sin la cual un fenómeno no podría comprenderse, es indispensable como tal. Estas situaciones son la que hacen posible que ciertos factores produzcan cambios importantes, es decir, determinantes, palabra que deriva de determinar que significa: "Fijar los términos de algo, 2. tr. Distinguir, discernir" (DRAE, 2001). Entonces, se trata de factores que en esas condiciones fijan los términos y explican el proceso ocurrido. Estos son factores determinantes. De este modo, se ha buscado entender este fenómeno identificando los factores condicionantes y determinantes.

Cuando los grupos de jóvenes conversaron en torno al abandono de los estudios y usaron para ello expresiones voluntaristas como por ejemplo: "Cuando dejé los estudios", "Cuando me retiré"; o como lo dicen los padres "el día que no quisieron ir más", inducen a pensar en que este problema es un fenómeno individual y se trata de una decisión propia.

El sistema educativo, por su parte, denomina este fenómeno de la exclusión como un acto de deserción, como el acto de abandonar las obligaciones o los ideales. Así el Diccionario de la Real Academia de la Lengua, en este caso, define naturalmente la metáfora de la deserción (DRAE, 2001). Para el sistema, entonces, son los estudiantes los que desertan, es decir, actúan como aquel soldado que abandona sus banderas (DRAE, 2001): El estudiante abandona sus ideales, y abandona el sistema que se los está proveyendo. Pero, ¿realmente se puede considerar voluntario el acto de dejar tirado los estudios, y por eso, tal vez, desistir de mejorar sus condiciones de vida? La autoría de este artículo piensa que no, que es una decisión condicionada y determinada por mecanismos de diverso tipo que operan como medios de exclusión. Sin saber, el estudiante o la estudiante se inculpan de ello: Ser pobre, asistir a una institución que no sabe cómo manejar la "deserción" y que no facilita el aprendizaje son condiciones insoslayables de exclusión.

No obstante, el concepto de exclusión no deja de ser también un concepto heterogéneo y confuso, por la multiplicidad de significados y de situaciones sociales a las que se hace referencia con él (Castel, 2004). Mas precisamente, señala este autor, desde una perspectiva crítica, la noción de exclusión solo tiene capacidad explicativa cuando la comprendemos como "desembocadura" de un proceso que conduce hacia un desligamiento, dada ciertas condiciones de vulnerabilidad previas (Castel, 2004). Así enfatiza que la exclusión es un producto social, marcado por la lógica de subprocesos vulnerabilizadores, tal como los continuos tránsitos de los jóvenes entre el ausentismo y la presencia en las aulas y las instituciones como pasos previos a su abandono definitivo, hasta alcanzar la cota más alta de exclusión de lo educativo. Sin embargo, esto no puede servir de base para pensar que se trata de un excluido total: un indigente. No, porque un grupo importante de estos estarán incluidos en el marcado laboral 
a corto plazo. Porque estos jóvenes son ahora trabajadores, están incluidos en el mercado laboral. La articulación entre exclusión educativa e inclusión laboral es un proceso social no observado con facilidad desde el Estado, porque las políticas sociales de inclusión, tales como los esfuerzos por retener en la institución educativa, no pueden reconocer este estrecho vínculo, aunque sí son capaces de denunciar el fenómeno específico de abandono escolar.

Así lo reconoció el Plan Nacional de Desarrollo “Jorge Manuel Dengo Obregón” 2006-2010 (Ministerio de Planificación y Política Económica [MIDEPLAN], 2007), al plantear que no todos los estudiantes realmente desertaban: “(...) no hay una única causa de la baja cobertura educativa y de la mal llamada deserción (...)"(p. 44), algunos son excluidos ${ }^{3}$, otros expulsados y otros repelidos (MIDEPLAN, 2007). Se coincide, por tanto, en que se trata de un fenómeno social complejo.

Como tal, este problema es objeto de preocupación de instituciones internacionales. La ONU se ha propuesto, como meta, al menos reducir este problema en primaria. Entre sus objetivos de desarrollo del milenio ${ }^{4}$ se plantea alcanzar una matriculación del 100\% en el 2015.

La consecución de esta meta resulta compleja, pues está relacionada, a su vez, con la meta de la reducción de la pobreza, que según el Informe 2008 (UN-ODM) de Naciones Unidas depende del acceso al empleo.

Al respecto, no se puede reducir la pobreza sin empleo pleno y productivo, sin trabajo decente para todos. La proporción de población en edad de trabajar que se encuentra empleada es un buen indicador de la capacidad de una economía para generar trabajo. Sin embargo, no existe una relación óptima entre empleo y población. La educación es un factor de desarrollo y de generación de empleo incomparable para alcanzar metas nacionales de calidad de vida y superación de la pobreza. La exclusión educativa es un problema que limita estas aspiraciones de desarrollo individual y crecimiento de la economía nacional, tal como señalan Jiménez, Robles y Arce (2009).

De acuerdo con los primeros aportes sobre la relación entre educación y crecimiento, la educación provee la plataforma para aumentar el nivel de habilidades de los trabajadores lo cual conduce, finalmente, a un mayor nivel de producción agregada. En otras palabras, estas contribuciones se basaban en la tasa de retorno de la escolaridad y su impacto sobre el crecimiento económico de largo plazo (...). (p. 204)

En América Latina el promedio de exclusión fue, durante el año 2003, de 37\%, es decir 15 millones de estudiantes adolescentes latinoamericanos de 15 y 19 años abandonaron los

3 Excluir significa"1. tr. Quitar a alguien o algo del lugar que ocupaba. Excluir a alguien de una junta o comunidad, 2. tr. Descartar, rechazar o negar la posibilidad de algo. Los datos excluyen una hipótesis contraria a ellos, 3. prnl. Dicho de dos cosas: Ser incompatibles". (DRAE, 2001)

4 Ver Naciones Unidas (s. f.). Podemos erradicar la pobreza 2015. Objetivos de desarrollo del milenio. 
URL: http://www.una.ac.cr/educare

CORREO: educare@una.cr

estudios, muchos de los cuales lo hicieron antes de terminar el ciclo básico. La mayoría, no obstante, lo abandona en el primer año de secundaria. Además 1,4 millones de niños y niñas nunca asistieron a la escuela (PREAL, 2003).

Después de este dato sintético para América Latina, las fuentes especializadas no lo han vuelto a calcular; no obstante, se tiene más actualizada la tasa neta de escolaridad para algunos países de la región: en el año 2008, un promedio de 68,12\%; en el 2009,67,32\% y en el 2011, $73,16 \%{ }^{5}$ (este último promedio solo cubre 5 países). La tasa neta implica un rezago importante de estudiantes en secundaria, ya que estos promedios explican a los estudiantes que no están cursando el año que les corresponde, y fueron los casos más sensibles de exclusión por rezago. Por otro lado, está el porcentaje de estudiantes que no estudian y trabajan, y de los que no estudian y no trabajan, estos últimos inclusos excluidos del mercado laboral. Así, para los años 2009 y 2011 , por ejemplo, el promedio de los jóvenes de entre 15 y 17 años que no estudian, pero trabajan era de $34,45 \%$ y $27,88 \%$, aunque en el 2011 los datos se refieren solo a 5 países. En cuanto a los jóvenes de esas edades que no estudian ni trabajan, el promedio en el año 2009 fue de $15,15 \%$, y para el $2011,12,98 \%$, aunque este último dato también incluye solo a 5 países ${ }^{6}$ (Sistema de Información de Tendencias Educativa en América Latina [SITEAL], s. f.).

Costa Rica no dista mucho de este diagnóstico regional. La exclusión escolar es un fenómeno persistente de la educación pública nacional, y afecta a un gran número de personas menores de edad, que deben abandonar el sistema educativo por razones de diversa índole, referidas más adelante. Este fenómeno es más profundo en la educación media (III y IV Ciclo) que en los ciclos anteriores. A continuación, un breve repaso:

Tomando como referencia el período 2001 al 2008, el abandono escolar, en el tercer ciclo de la académica diurna, rondó por el 12\%, mientras que en la nocturna fue de más de $24 \%$ (Ministerio de Educación Pública. Departamento de Análisis Estadístico, 2009). Por regiones, en el año 2008, la exclusión fue de un 12,1\% en total, y la Dirección de Regional de Educación de Aguirre presentó el dato más alto, luego esto se dio en Turrialba con un 6,4\%7 (MEP. Departamento de Análisis Estadístico, 2009).

5 En el 2008 el cálculo se realizó con base en Argentina, Brasil, Costa Rica, República Dominicana, Ecuador, El Salvador, México, Paraguay, Uruguay y Venezuela. En el 2009, además de los anteriores, Bolivia, Chile, Honduras, Nicaragua, Panamá, y en el 2011 participaron solo Argentina, República Dominicana, Panamá, Paraguay, Uruguay y Venezuela.

6 Jóvenes que en el año 2009 no estudiaban pero trabajaban: Argentina, Bolivia, Brasil, Costa Rica, Chile República Dominicana, Ecuador, El Salvador, Guatemala, Honduras, Nicaragua, Panamá, Paraguay, Perú, Uruguay y Venezuela; y en el 2011 solo se cuenta con el dato para Argentina, Republica Dominicana, Guatemala, Paraguay y Uruguay. Y en el caso de jóvenes que no estudiaban ni trabajaban en esos mismos años. Se tienen, básicamente, a los datos de los mismos países.

7 Costa Rica 12,1, San José 9,4, Desamparados 11,1, Puriscal 11,4, Pérez Zeledón 12,3 , Alajuela 10,3, San Ramón 12,0, San Carlos 15,0, Upala 15,5, Cartago 11,9, Turrialba 6,4 , Heredia 9,3, Sarapiqui 9,3, Liberia 15,4, Nicoya 11, Santa Cruz 12,4, Cañas 15,2, Puntarenas 13,0, Coto 17,9, Aguirre 20,1, Grande de Térraba 13,8, Limón 15,3, Guápiles 14,9. Elaboración propia con base en datos proporcionados por el Dpto. de Análisis Estadístico, MEP. 
Por otra parte, concentrando la atención en las ofertas académicas y diurnas ${ }^{8}$, fue la Dirección Regional de Educación de Liberia la que presentó mayor abandono en la modalidad de educación técnica diurna, mientras que en la académica diurna fue Upala.

Si se analiza solamente la oferta académica diurna se observa, como dato interesante, que las ofertas educativas de colegios científicos y de bachillerato internacional son las que presentaron, en ese año, los mayores porcentajes de abandono $-15,3 \%$ y $15,8 \%$ respectivamente-, seguidas por las telesecundarias 13,3\% (MEP, Departamnto de Análisis Estadístico, 2009)9.

Las modalidades de académicos diurnos con menor deserción fueron la del colegio humanístico y de los experimentales bilingües, en ambos casos la pedagogía utilizada tiende a ser muy distinta, mucho más constructivista y de desarrollo de la criticidad del estudiante ${ }^{10}$. Y es sétimo el nivel con mayor abandono.

A modo de ejercicio respecto de esta problemática, se muestra cómo el abandono escolar medida por cohortes es bastante numeroso: alrededor de 50000 personas abandonaron los estudios en el periodo 2001-2005 (de un total matriculado en el 2001 de 86497), 53000 en el período 2002-2006 (la matrícula en el 2002 fue de 91326) y 55000 en el periodo 2003-2007 (de una matrícula en el año 2003 de 95380). Estas cantidades de personas son la diferencia ente matricula inicial y final de los años del periodo de los ejemplos. Son muchas personas, ¿no? Es en el mismo Plan Nacional de Desarrollo 2006-2010 (MIDEPLAN, 2007) donde lo observa:

(...) Costa Rica mantiene a dos terceras partes de sus jóvenes por debajo de ese piso: no logran terminar la secundaria. De esta forma, la incapacidad del sistema educativo provoca tanto un impacto negativo sobre el potencial de crecimiento del país (...) pero provoca un impacto aún más peligroso en los procesos de integración social, ya que solamente abre oportunidades de un trabajo y una vida mejor para una tercera parte de la población, excluyendo de esas oportunidades (...) al resto. (p. 44)

Estamos lejos de la cobertura del $100 \%$. Tal como se señala en el Plan Nacional de Desarrollo (PND), cuando plantea la necesidad de alcanzar una cobertura (MIDEPLAN, 2007), ya que hay abandono de estas ofertas.

8 Con porcentajes de deserción de 12,2 y 10,9\% respectivamente (Ministero de Educación Pública. Departamento de Análisis Estadístico, 2009).

9 El comportamiento en las otras modalidades es como sigue: Humanístico: 4,8; Liceo rural: 5,1\%; Exp. Bilingüe: 6,6\%; IEGB: 8,5; Ambientalista: 8,9\%; Deportivo: 9,4\%; Orientación tec.:11,3\%; Liceo Modelo: 11,8\%; Artístico: 13\%; Valor Agregado: 13,6\%. (Ministero de Educación Pública. Departamento de Análisis Estadístico, 2009).

10 Cabe destacar que para ingresar a ambas modalidades se escoge a los estudiantes mediante pruebas de admisión o por promedios de calificaciones obtenidas durante su educación primaria. 
URL: http://www.una.ac.cr/educare

CORREO: educare@una.cr

Los testimonios de las familias, conocidas en esta investigación, hablan de factores que determinan el abandono escolar: factores de orden económico, social y educativo, que operan sobre condiciones dadas como la pobreza y la desigualdad social y sobre la falta total de pertinencia educativa.

\section{Antecedentes}

Una de las primeras referencias en materia de estudio se encuentra en el año 1956, cuando don Rafael Cortés, de la Escuela Pedagógica de la Universidad de Costa Rica, realiza una monografía titulada La deserción escolar en la que reúne datos de deserción de la década de los treinta hasta la de los cincuenta. En este estudio se señala la deserción como un "problema fundamental de la democracia costarricense (...) es de los más conocidos y trajinados por los maestros y funcionarios de educación" (Cortés, 1956, p. 3).

El breve estudio de Cortés -el que no por ello deja de ser una detallada recopilación de datos, de fuentes oficiales de la época, que le permiten reconstruir el problema desde los años 30 en adelante- muestra cómo en Costa Rica, en cincuenta años de educación gratuita y obligatoria, solo el $14.69 \%$, de todos sus ciudadanos han alcanzado la primaria completa. Y en los años 50 con un población de 618977 personas, terminaron la primaria solo el 8,51\% y secundaria 1,19\% (Cortés, 1956). De los años anteriores, estudiados por este, las tasas de deserción son parecidas.

Luego de este remoto antecedente, se han realizado a través de los años nuevos estudios, la mayoría de ellos investigaciones básicas centradas en la ocurrencia de este fenómeno en distintas instituciones educativas. Estudiadas estas, como casos particulares, han generado diversas explicaciones para comprender el fenómeno de la deserción.

Diez años después de este "remoto" antecedente, aparece el estudio de la señora Fajardo Rendimiento académico, retención y deserción escolar, desde 1963, y estudio de algunas características de los alumnos de IV y V de 1967 del Liceo Mauro Fernández A. (1968), con una metodología que combinó un enfoque cuantitativo con uno cualitativo. El estudio se basó en cuestionarios, observaciones y entrevistas a 125 estudiantes del Liceo Mauro Fernández. En ese entonces definió “Deserción" como: "(...) el abandono que por diferentes motivos hacen los estudiantes de las aulas de cualquier institución de enseñanza (...)" (Fajardo, 1968, p. 9).

En él indica que:

Debido a la concepción académica que rige en el régimen de enseñanza, a factores geográficos, sociales y demográficos -no investigados plenamente se produce en todo el sistema educativo una deserción sistemática de los alumnos en todos los niveles inferiores, tanto de la enseñanza primaria como de la enseñanza media. Revela pues cierta anormalidad del sistema. (p. 27) 
Plantea que la deserción es alarmante, sobre todo en los tres primeros años "(...) acentuándose [sic] considerablemente en el paso de primer a segundo año (...) siendo [sic] los varones más que las mujeres los que abandonan las aulas" (Fajardo, 1968, p. 27-28). Sugiere como casusas: "el cambio de domicilio, la situación económica, la indiferencia del hogar y la enfermedad y otras" (Fajardo, 1968, p. 33). Causas parecidas a las identificadas en otros estudios.

Entre los años de 1998 y el 2002, varios trabajos de graduación de la Universidad de Costa Rica (UCR) tuvieron como tema la deserción escolar.

En esta dirección, Loría, Rodríguez y J. M Ureña y J. B. Ureña (1998), en su tesis de licenciatura El rendimiento académico y la deserción de los estudiantes en el proceso de transición de sexto a sétimo año de la Educación General Básica, en el circuito escolar 06 de la Dirección Regional de Enseñanza de San Ramón en el período 1996, se interesaron en la búsqueda de explicaciones de la deserción en la transición de sexto a sétimo año y en las razones para explicar la ocurrencia de este fenómeno en ese momento de transición.

Rojas (1998) se preocupa acerca de la inducción al cambio en sétimo entre los estudiantes del Colegio Mario Quirós Sasso. La deserción en la transición de sexto a sétimo, ubicada en los Colegios Julio Acosta, Técnico de Pacayas, fueron los temas de Pérez (1999), de Irola (2002) y del trabajo de Barrantes (1999) de apoyo a la transición hacia el colegio.

En todos estos trabajos de investigación se identificaron, básicamente, los mismos factores: falta de escolaridad de los padres, la profesión u oficio de estos, el desinterés de los padres de familia, el bajo rendimiento académico, desmotivación, problemas económicos, problemas familiares, problemas de salud, necesidad de trabajar, cambio de domicilio, problemas con los docentes, temor a los exámenes, fallas pedagógicas y falta de una transición adecuada a sétimo. Ninguno de estos estudios se enfocó causalmente, sino que se interesaron en establecer factores responsables (Loría et al., 1998).

Estos autores, definen el fracaso escolar como "(...) dificultad grave que puede experimentar un niño, con un nivel de inteligencia normal para seguir un proceso escolar de acuerdo a su edad (...)" (Loría et al., 1998, p. 62) y mantienen la sospecha de que este sea un factor clave que puede redundar en deserción, entendida, también, como el "(...) retiro que hace un niño de las instituciones educativa de enseñanza formal (...)" (Loría et al., 1998, p. 62), con lo cual siguen la tradición culpabilizadora de los estudiantes. Agregan que "El alumno y el padre de familia dicen que la desmotivación es la que hace desertar al primero; los docentes anotan que el rendimiento académico y los problemas económicos son los elementos más sobresalientes causantes de tal situación" (Loría et al., 1998, p. 142).

Estableciendo una serie de preguntas causantes de la deserción escolar en I y II Ciclo, referidas a la responsabilidad de la situación económica, inadecuadas relaciones familiares y las medidas tomadas por el docente para enfrentar el problema, Jiménez, López, Saborío y Solano (1997) se abocan a la tarea de efectuar un análisis de los factores que causan deserción y establecen una clasificación de las causas en tres tipos: problemas escolares, problemas 
URL: http://www.una.ac.cr/educare

CORREO: educare@una.cr

económicos y problemas familiares; detallaban los entrevistados de 6 instituciones de Puntarenas que las causas eran los problemas de ayuda por parte de la familia, el desinterés de los padres de familia, trabajo, falta de materiales, problemas en la comprensión de la materia, muchas peleas con los compañeros, problemas económicos, bajo rendimiento académico y problemas sociales.

Hernández y Barrantes (2002), investigadores del Ministerio de Educación, del Departamento de Estudios e Investigación Educativa, realizan el estudio Deserción escolar en sétimo año. Factores que inciden en la deserción escolar de estudiantes de sétimo año. En este, la selección de sétimo obedeció a las mismas razones que buscan hoy día explicar el mismo fenómeno., las autoras definen la deserción como "abandono que hace el estudiante de sus estudios de educación secundaria en el sistema formal de enseñanza" (Hernández y Barrantes, 2002, p. 35), o sea, abandono del colegio, aunque haya continuado en otras opciones del sistema no formal. Esta última definición, de modo muy sutil, hace descansar la responsabilidad de este fenómeno, de nuevo, en el estudiante y es en la que concuerdan la mayoría de los autores revisados. Al finalizar el estudio concluyen que los principales factores son: Problemas económicos, de rendimiento académico, programas de estudio que no responden a las necesidades del estudiante, desinterés por el estudio, problemas de aprendizaje, carga académica, exámenes memorísticos y la metodología utilizada por el docente (Hernández y Barrantes, 2002).

Más recientemente, el trabajo de Benito (2005) La exclusión escolar desde la percepción de los (as) niños (as) y adolescentes se concentra en la percepción de la deserción por parte de un grupo de niñas de barrios marginales. Buscó conocer las percepciones de estas niñas respecto de la deserción, la visión subjetiva de este fenómeno. Luego de repasar los vacíos de información existentes al respecto, se inclina por un estudio desde la subjetividad en el que se superen algunos equívocos como los de achacar sin más y por diversos planteamientos la culpa a los estudiantes y las estudiantes (Benito, 2005). Señala que la exclusión escolar tiene efectos negativos en la vida de las personas a mediano y largo plazo en cuanto a ascenso social, de construcción de ciudadanía, acceso a empleo digno y al desarrollo de una mejor calidad de vida (Benito, 2005).

Los factores de deserción que identifica, al igual que los otros autores, son: las condiciones económicas y sociales, la diversidad individual y familiar, la comunidad en la que se desarrollan, las relaciones con otros niños y adolescentes y las características del sistema educativo en que se han desenvuelto las participantes (Benito, 2005). Factores que se ven también influenciados por algunas condiciones tales como la infraestructura, el presupuesto, y las metodológicas, las aptitudinales, tanto de docentes como de estudiantes (Benito, 2005), y el rol del grupo de pares, porque genera expectativas positivas o negativas hacia el proceso de formación, "situación que puede redundar en incrementar el riesgo de exclusión escolar" (Benito, 2005, p. 140).

Como se observa, los estudios revisados y muchos otros plantean débilmente algunas relaciones de causalidad entre los factores y sus consecuencias, y no se puede detallar hoy, con claridad y para una gran mayoría de los casos, cuáles son los factores más determinantes, solo hay tímidas aproximaciones. 
En síntesis, la mayoría de los estudios coinciden en los factores asociados a la deserción o exclusión social y en el carácter multicausal de este fenómeno, que implica, como tal, el diseño de medidas y decisiones de política pública orientadas a la intervención en la diversidad de factores determinantes identificados como los de índole económica, social, cultural y pedagógica.

Este conjunto de factores se repiten en la bibliografía revisada y, probablemente, todos tienen un peso importante en el proceso de gestación de fenómeno de deserción; no obstante, para intervenir es necesario hacer estudios más específicos que tengan por objetivo determinar la importancia relativa de unos factores por sobre otros. Desde el punto de vista del Ministerio de Educación, es necesario establecer la importancia de los factores en donde se puede intervenir institucionalmente o sugerir intervenciones a otras instancias.

\section{Metodología de la investigación}

La investigación se desarrolló a partir de un enfoque cualitativo y naturalista para acercarse a este fenómeno, con el propósito de recuperar la percepción directa de los diversos actores de la problemática en estudio, en este caso, de la comunidad educativa, principalmente de estudiantes que hayan abandonado el colegio, estudiantes regulares, padres y madres y madres de familia, orientadores y directores.

En particular, interesó recuperar la voz de los jóvenes y los significados que estos le asignan al proceso vital que experimentan y que los conduce al camino del abandono escolar y a la exclusión educativa. Estos significados son importantes para ensayar una interpretación del fenómeno de la "deserción" fundada en la percepción local del fenómeno del abandono escolar.

La población de este estudio se constituyó por los estudiantes de secundaria de todo el país que estuvieran matriculados en ese año 2009, y que hubieran estado cerca de abandonar el colegio; y un grupo de estudiantes que efectivamente los hubiera abandonado en forma definitiva. También se consideró importante conversar con los padres de familia, con orientadores, docentes y directores institucionales.

El estudio se realizó en 22 colegios públicos pertenecientes a todas las regiones educativas del país. Entre las características relevantes de este grupo de colegios seleccionados sobresalen las siguientes: a) Reportaron altas y bajas tasas de deserción en el año 2008, b) Se trata de colegios urbanos y rurales, c) Las instituciones representan la mayoría de las ofertas educativas (académicos diurnos, telesecundarias, colegios técnicos, indígenas, institutos de educación general básica (IEGB) y artísticos. Los colegios nocturnos no fueron tomados en cuenta en este estudio, por las características adultas de la mayoría de la población y por la alta tasa de deserción de esa oferta. Por esa misma razón, tampoco se consideraron las ofertas de educación abierta. 
URL: http://www.una.ac.cr/educare

CORREO: educare@una.cr

En cuanto a la metodología de producción de datos, se constituyó por medio de la aplicación de 22 entrevistas (orientadores y directores) y 22 grupos focales (estudiantes y docentes), visitas a a padres y familia de estudiantes de $13^{11}$ de los 22 colegios participantes, (colaboró un promedio de 3 padres, madres y encargados por grupo focal -aunque se trató de que fueran al menos 10, la convocatoria por parte de las direcciones no fue la esperada-). La sistematización y análisis se realizó por medio de los procesos de codificación y categorización de contenidos, de acuerdo con las siguientes categorías: las decisiones personales, el rol de la familia, la pertinencia de la gestión educativa institucional, la vida social de los estudiantes y las condiciones sociales y económicas.

Por otra parte, las guías de producción de datos que se usaron en las entrevistas y grupos focales se ordenaron de acuerdo con un set de indicadores que orientaron el desarrollo de las actividades de campo, estos fueron: 1) Cobertura estadística por región, nivel y sexo. 2) Fases de la deserción. 3) Razones de deserción: tipos de razones, valoraciones de la educación, importancia dada a la educación. 4) Tipos de apoyo familiar: características, tipo de familia, apoyos que brinda. 5) Situación social de la familia: escolaridad de los miembros de la familia, facilidades de estudio en la casa, valoración del estudio, responsabilidades académicas de los padres, número de miembros. 6) Situación económica de la familia: ingresos, egresos, gastos en colegios. 7) Relación institucional: características, tipo de atención brindada, percepción institucional del estudiante. 8) Factores de retención dentro del sistema educativo.

La ordenación y sistematización se realizó, como es costumbre, por medio de matrices de doble y triple entrada, en las que la información se ubica con relación con las categorías e indicadores de la investigación, de tal modo que se evidencien sus cruces y patrones. Estos componentes están soportados por los objetivos específicos.

En particular se ha puesto en práctica, para la sistematización de los datos, el método de comparación constante (MCC) (Flick, 2004), que facilita la ordenación teórica de una masa importante de información cualitativa, por medio de procesos recurrentes de codificación y categorización de la información, hasta llegar la formulación de categorías comprensivas del fenómeno que se estudia a partir de los planteamiento realizados por los informantes. Se apoya fundamentalmente en esta metodología, que es hija del enfoque conocido como teoría fundada (Grounded Theory) y también en programas de cómputo como el Atlas Ti, usado en esta investigación para la sistematización de los datos.

\section{Estudiantes y familias, en el borde del fracaso. Conceptualización de los resultados}

Los resultados logrados mostraron que los factores causales para los distintos actores de la comunidad educativa se resumen en una compleja trama entre la situación económica y social de pobreza relativa en la que viven la mayoría de los jóvenes y las jóvenes que abandonan los estudios: la vida social y sus imperativos; una gestión educativa inadecuada por

11 Instituciones de las regiones educativas de Upala (Zona Norte-Norte), Pérez Zeledón, Heredia, Coto, San Carlos, San Ramón (hoy Occidente), San José, Santa Cruz, Sarapiquí, Puntarenas, Cañas y Limón. 
parte de las instituciones, que involucra directores, docentes y orientadores; la insuficiencia de los programas de equidad, y la pobreza de los grupos familiares, que no tienen tampoco la capacidad educativa de ayudar a sus hijos e hijas.

El contexto económico y social del grupo de jóvenes de las instituciones públicas de secundaria visitadas, está caracterizado por la presencia de situaciones de pobreza y carencias para atender las necesidades básicas. Independientemente de la naturaleza rural o urbana, la condición étnica, la realidad portuaria de uno u otro colegio, o la modalidad de estos (académica, técnico o artístico), en la opinión del grupo de jóvenes entrevistados y de los funcionarios con los que se tuvo contacto, la situación de pobreza existente se vuelve determinante de las decisiones acerca de abandonar los estudios. Contextos en los cuales las salidas laborales son, en muchos casos, sus opciones reales, ya sea para ayudar a la familia o para ayudarse a sí mismos.

A parte de las ayudas brindadas por el programa Avancemos -que en términos generales es bien valorado-, para enfrentar el problema del abandono escolar, los estudiantes no identifican otras soluciones en el campo educativo, respecto de cuya gestión plantean muchas quejas; pero no detallan soluciones.

El abandono de los estudios implica, por lo general, una salida laboral; salida que es una opción cercana a los jóvenes de edad y grados más avanzados, más que para los de sétimo y octavo, quienes no tienen otra opción que quedarse en la casa de "vagos".

El estudiantado no visualiza las implicaciones negativas que a largo plazo pueda tener la decisión de abandonar; no obstante, muchos guardan la esperanza de ir a la universidad y de tener un mejor futuro, es decir, ascender socialmente.

El abandono parece ser un asunto personal, una decisión que es tomada por diversas razones, después de evaluar su situación económica familiar, o bien, la educativa, o una combinación de ambas. Otros estudiantes abandonan porque educacionalmente no les va bien, han fracasado en los exámenes y su rendimiento es bajo. Estos, sin embargo, no parecen ser tan individuales. Hay razones para pensar de este modo. Por otro lado, se trata de situaciones ante las cuales las instituciones educativas hoy no pueden hacer mucho por falta de condiciones y competencias específicas para prevenir y retener.

En tres dimensiones se han buscado, generalmente, las explicaciones de este fenómeno: el rol de la familia, el del sistema educativo y el económico laboral. A continuación revisaremos los datos obtenidos con una mirada más amplia.

\section{El rol de la familia}

La importancia de la familia en los procesos educacionales de los hijos es persistentemente citada como factor que explica el abandono escolar. No es el factor que lo determina, pero sí lo condiciona. 
URL: http://www.una.ac.cr/educare

CORREO: educare@una.cr

En este sentido, es más determinante la condición socio-económica de la familia que el tipo de estructura de esta. Así que para que un adolescente termine sus estudios, desde el ingreso en primaria hasta su finalización en secundaria, se requiere, según el informe del Sistema de Información de Tendencias Educativas en América Latina (SITEAL) (2008), de un gran esfuerzo familiar y para ello se debe contar con un nivel mínimo de bienestar, que haga posible dar, la prioridad que merece, a la educación de estas nuevas generaciones

Este bienestar permite que los alumnos concurran bien alimentados, saludables y descansados a clases, que puedan dedicar la mayor parte del tiempo, durante años, a su educación, y que no recaigan sobre ellos responsabilidades que tienen que ver con la supervivencia y el funcionamiento básico de sus hogares (p. 43).

Estas responsabilidades, relacionadas con la articulación de las familias al mercado laboral, constituyen el punto de partida para abordar el tema del apoyo familiar que se brinda a estudiantado y ponen al descubierto un componente estructural, que determina que los mayores problemas de las familias tienen relación con situaciones de pobreza y exclusión social, donde los cambios en el mercado de trabajo son una prioridad, debido a:

1. Incorporación de las mujeres al mundo asalariado.

2. Aumento del trabajo informal y la desocupación, aún en el marco del crecimiento económico.

3. Mayor fragmentación social por muchas razones, entre ellas, la menor cantidad de puestos de trabajo que se generan solo son accesibles a las clases con mayor capital social.

4. Precarización de las relaciones laborales, por el crecimiento del sector informal. Los que no encuentran trabajo en el sector formal crean ocupaciones estructuradas, según la lógica de la supervivencia y son de carácter precario e inestable.

5. Violencia resultante de los crecientes diferenciales de ingresos y de la frustración ciudadana (Picaroni, 2009, pp. 87-88).

Cuando los padres de familia tienen una vinculación muy débil con el mercado de trabajo, en tareas de baja remuneración o posiciones inestables, el resto del hogar queda comprometido en acciones orientadas a generar ingresos. Esto se refleja en una mayor participación de los adolescentes en el mundo del trabajo y en una tendencia pronunciada a conformar hogares ampliados, en los que la convivencia con otros familiares o no familiares 
representa una estrategia solidaria de reducción de costos y construcción conjunta de la base de ingresos. Todos estos factores ayudan a configurar, en cada familia, el escenario en que los niños y adolescentes deben afrontar el esfuerzo que representa su educación (SITEAL, 2007).

En estos casos se reproduce un círculo de pobreza económica que se expresa en menor cantidad de recursos para invertir en la educación de sus hijos y, por ende, menores oportunidades para insertarse laboralmente.

En distintos estudios se ha encontrado que la fecundidad de las mujeres con escasa educación formal suele iniciarse más temprano y resulta en hogares conformados con un mayor número total de hijos y con mayores dificultades para afrontar los costos de mantenerlos en el sistema escolar, por lo tanto, con menor disponibilidad de recursos económicos para sus necesidades personales, menor acceso a la atención personalizada de los padres y una inserción más temprana al mercado de trabajo para así aportar ingresos al presupuesto familiar o ayudar en la casa para apoyar el trabajo remunerado de la madre (Geldstein, Loayza, Fukazawa, Kaztman y Feijoó, 2006).

En el informe de SITEAL (2008), al respecto, se señalan algunas de estas condiciones que agravan la problemática educativa. En concreto, este informe se refiere al hecho de que en las familias pobres de Centroamérica no solo "(...) hay más hogares con adolescentes, sino que además hay más adolescentes por hogar. Esto responde a una estructura poblacional muy diferente, que resulta de sociedades con mayor presencia de niños y adolescentes, y menor presencia de adultos mayores (...)" (SITEAL, p. 36). Esta realidad es más fuerte "(...) en las zonas rurales que en las urbanas (SITEAL, p. 45).

La falta de oportunidades de empleo para los padres, las prioridades impuestas de consumo y la brecha existente entre las expectativas altas de estudio por parte de los padres con la realidad compleja de problemas académicos y la inversión en educación visualizada como un gasto más en la familia hacen que se replanteen la educación de los jóvenes como una prioridad en el imaginario de estas personas, pero existe una realidad que se contrapone a la ilusión. En este sentido, los problemas económicos van acompañados de una cultura familiar en la que se manejan bajas expectativas con respecto al estudio "(...) gente sencilla, humilde, de muy escasos recursos económicos y que no tienen claro por qué estudian" (Entrevista con la directora, Colegio Técnico Profesional de Puerto Viejo, 2009).

De este modo, y por lo visto en la investigación llevada a cabo, la racionalidad familiar en relación con la problemática del abandono escolar se articula alrededor de dos ejes complementarios: 1) las prioridades familiares partiendo de sus dinámicas internas y 2) los recursos que puedan destinar estas familias al área educativa.

Por otra parte, fue evidenciado por este estudio que la condición económica baja de las familias contribuye a establecer dinámicas de sobrevivencia que muchas veces irrumpen en la estabilidad emocional de los jóvenes por la inclusión o exclusión continua de alguno de los miembros de la familia; la disfuncionalidad entendida como madres de familias con más de 
URL: http://www.una.ac.cr/educare

CORREO: educare@una.cr

un compañero, en donde hay choques de los jóvenes con sus padrastros, y en un ambiente en donde también hay problemas de hacinamiento (Entrevista con director, Liceo Ricardo Fernández Guardia, 2009).

Estas estructuras familiares aún no son entendidas por el centro educativo, el cual promueve una educación dirigida a familias tradicionales en contextos modelos -ese estudiante integrado, de sectores medios altos y urbanos que se alejan mucho de la realidad de estos estudiantes-. En este sentido es fundamental explorar la pertinencia de la educación que actualmente se ofrece; en otras palabras: hay que reestructurar la promesa educativa que el sistema brinda a los jóvenes.

En síntesis, la familia como entidad no puede tener un efecto positivo en el éxito de los estudiantes y, por ese medio, evitar el abandono, pues esta está determinada por sus modos de inserción económico y mercados laborales a partir de los cuales, como colectivo, genera los recursos para la atención de las necesidades básicas, circunstancia que, en general, requiere muchas veces del aporte de las mayor cantidad de sus miembros, incluidos los niños y los adolescentes, los que deben para ello abandonar sus estudios, suspenderlos o posponerlos.

En una cantidad importante de las familias entrevistadas, los programas de equidad tienen resultados positivos, como se vio con los testimonios que indicaban que sin la beca del IMAS o el aporte del comedor y del transporte no podrían estudiar; mientras que para otro número creciente de familias estos aportes resultan a todas luces insuficientes. A modo de hipótesis se sugiere que estas contribuciones son insuficientes cuando ese ingreso de la beca del IMAS es el principal -que es lo observado en algunas de las familias entrevistadas-.

\section{La gestión educativa institucional}

Otra de las razones que fundan la decisión de abandonar el colegio la ubicamos en el campo educacional. Los estudiantes, principalmente, y algunos directores y orientadores consultados manifiestan que la baja en el rendimiento académico es la principal causa de deserción. Esta se verifica, como ya se ha señalado, en el tercer trimestre, después de vacaciones de medio año, después de constatar las malas notas en el primero y en el segundo.

Pero, ¿por qué un estudiante obtendría malas notas, por qué tantos estudiantes obtienen malas notas $y$, coincidentemente, por qué les sucede esto a los más pobres, en forma mayoritaria?

Aquí se abre una discusión acerca del currículo educativo, el cual en una primera impresión y a partir de la opinión de los jóvenes aparece como si este no fuera pertinente. Es decir, las opiniones de los jóvenes nos ubican en un currículo que no les gusta, es aburrido, está mal gestionado pedagógicamente, es muy vertical. En síntesis, es inadecuado y en muchos casos es el que propicia la deserción. 
No obstante, lo que es relevante comprender es que al parecer el currículo no es pertinente para aquellos que abandonan los estudios, por eso desertan. $Y$ no es pertinente, porque el joven no cabe dentro del concepto de estudiante que este plantea; concepto que, en cierto modo y a lo largo de un período de 12 años o más, el sistema educativo ha venido modelando para que los estudiantes se amolden al currículo, y no al contrario.

Esto obedece, según SITEAL (2008), a que los sistemas educativos han sido, en gran medida, exitosos para llegar a los adolescentes urbanos de las clases medias y altas. En todos los países, los adolescentes de los sectores urbanos más integrados muestran una escolarización elevada, niveles de retraso bajo y altas tasas graduación en la escuela secundaria "(...) Las grandes diferencias que se perciben entre los países en sus logros educativos responden fundamentalmente a cuán extensas son esas clases más integradas y qué capacidad tiene cada país de incorporar a las prácticas escolares a aquellos otros sectores sociales más postergados (...)" (p. 87).

Y amplía SITEAL (2008), señalando que:

(...) hay una gran dificultad en la región para crear las condiciones sociales, culturales y educativas orientadas a que todos los adolescentes estén escolarizados y logren completar el nivel medio. Esto se aprecia en los datos que muestran la situación de aquellos que viven en zonas rurales, los de familias afrodescendientes o indígenas y los de nivel socioeconómico más bajo. Sin duda, ante estos grupos sociales los sistemas educativos muestran su debilidad y evidencian sus limitaciones (p. 87).

Esto, por cuanto la enseñanza secundaria está determinada por:

(...) la marca de origen que llevan estas instituciones: la educación media no fue concebida en los países de la región como una educación universal. Por el contrario, desde su origen, la oferta educativa en educación media estuvo orientada precisamente hacia los sectores urbanos más integrados y pesa sobre ella una larga tradición, un modo de relacionarse con el alumno y la comunidad y una propuesta institucional y pedagógica que logra sus mejores resultados en esas clases urbanas más acomodadas (SITEAL, 2008, pp. 87 y 91).

A la par de esta determinante histórica, existe un problema pedagógico relevante respecto a la gestión del currículo y la percepción de este por parte del alumnado, cuya respuesta es el ausentismo a clases. Algunas evidencias de este procedimiento simbólico se encuentra en los planteamientos realizados al respecto por parte de los directores y orientadores que fueron entrevistados. Manifiestan que los estudiantes y las estudiantes deambulan insistentemente 
URL: http://www.una.ac.cr/educare

CORREO: educare@una.cr

por el colegio, sin entrar a clases, y no se toman medidas en la institución para ver por qué no entran, o cuando los estudiantes hablan de clases aburridas, o de profesores que dictan, o que hablan, y hablan, y no explican.

Tal proceso de ausentismo genera problemas crecientes en el rendimiento académico del estudiante, lo dicen ellos mismos, luego vienen las malas notas y el abandono o el retiro de estos por parte de los padres de familia. Estas son prácticas docentes que obligan a la persona a adaptarse, sino queda excluida. La educación no es para ella.

De este modo, entonces, las decisiones personales no lo son tanto: las personas se ven compelidas a abandonar, ya que no calzan en lo que se espera de ellas. La percepción que tienen los estudiantes de la gestión educacional es muy negativa.

El problema central que se deriva de la percepción de la gestión educativa es que esta resulta definitivamente inadecuada respecto a los gustos, intereses y necesidades de los estudiantes. Así se leen en los testimonios de los estudiantes varios apelativos que definen con suma claridad esta percepción: clases aburridas; materias inútiles; sistema promoción que no sirve, sistema de evaluación injusto; docentes que no entienden a los estudiantes, que no comprenden sus necesidades ni la época de la adolescencia; régimen disciplinario autoritario (usan las boletas para todo).

Tales percepciones la ratifican, también, los directores institucionales y los orientadores, quienes paradójicamente son los que deben confeccionar y enviar las boletas disciplinarias. De estas percepciones se derivan consecuencias y decisiones. La consecuencia primera es la del ausentismo, de ahí se sucede el bajo rendimiento y, finalmente, se decide la deserción, la cual, una vez dada no recibe seguimiento por parte de la institución.

\section{Los factores económico-laborales}

En cuanto a la causalidad económica de esta problemática, son claros los testimonios de padres y madres de familia, estudiantes e incluso directores y orientadores acerca de los problemas diarios experimentados por las familias para satisfacer sus necesidades y atender sus condiciones de vida; situaciones que en muchos casos no se pueden resolver con los aportes de los programas de equidad, particularmente los del programa de Avancemos. Se requiere mucho más que eso.

Esta situación no ha sido solo observada por las familias consultadas, es también una preocupación nacional e internacional. Detrás de las razones individuales y familiares, subyace una fuerte determinación de las políticas económicas, sociales y educativas y, en ese marco, el papel central del Estado como proveedor de servicios educativos de calidad para todos (Jacinto, 2009) para lo cual justamente se han modelado: 
(...) grandes programas de prestaciones mínimas, esencialmente de becas, que exigen como contraprestaciones la asistencia a la escuela y la atención de la salud, y en algunos casos, buenos resultados escolares. Y por otra parte, se observan acciones que cuestionan el modelo institucional excluyente de la escuela, planteando que es preciso un mayor énfasis en las condiciones para generar mejores aprendizajes en la escuela regular y mayor diversificación institucional. (p. 3)

Estos programas deberían al menos contribuir también en la evitación del trabajo infantil y adolescente, orientado no solo a la contribución en la economía familiar, sino a la satisfacción de diversas necesidades y consumos de los adolescentes. Las salidas laborales son, como lo señalaron los mismos jóvenes, una de las principales razones por las que se abandona el colegio. Esta situación es una constante en los estudios de los sistemas educativos, cuando se refieren a los problemas que los aquejan.

El informe de SITEAL (2008) alerta de un fenómeno que afecta centralmente las posibilidades de éxito académico de los adolescentes, este consiste en la precarización del empleo:

(...) Informalidad y precariedad se traducen inequívocamente en ingresos insuficientes y, por ende, además de generar una mala situación económica general, son factores de presión para que los adolescentes se transformen en trabajadores. En resumen, gran parte de los hogares con adolescentes en la región viven en una situación de bajos ingresos, inestable y sin cobertura frente a las vicisitudes del ciclo vital, lo cual evidencia una vulnerabilidad objetiva de las posibilidades de mantenimiento en el sistema escolar de los adolescentes más desfavorecidos. (p. 46)

\section{Y agregan que}

(...) la expectativa creciente de que todos los adolescentes logren completar la educación secundaria, haciendo un buen aprovechamiento de esa experiencia para acceder a una educación de calidad, entra en conflicto con una situación de hecho: un tercio de ellos ya está integrándose, desde temprana edad, al mercado de trabajo (...). (SITEAL, 2008, p. 48)

Por otro lado, aparece también una problemática adicional, referida a los adolescentes que no estudian y no trabajan. Aquellos que no tienen como insertarse en un mercado laboral cada vez más exigente, y que salieron expulsados del sistema educativo por la falta de pertinencia de este.

Esta categoría comprende la proporción más importante de adolescentes sumidos en la marginalidad extrema de la que indudablemente se nutren el desinterés y la apatía, el delito, la violencia, las pandillas, las maras, las adicciones, la locura y la rebeldía sin cauce que se expresa como autoagresión o violencia hacia los otros. 
URL: http://www.una.ac.cr/educare

CORREO: educare@una.cr

Los desafíos de cobertura, calidad y pertinencia de la educación secundaria, para el amplio espectro de estudiantes de todas las clases sociales, y no solo de los urbanos de sectores medios y altos, exigen una educación pertinente -social y culturalmente-, lo que a su vez obliga alcanzar niveles de equidad relevantes y determinan que la acción educacional:

(...) se orienta a la reducción de brechas de acceso como las derivadas de condiciones de como lugar de residencia, sexo, etnia o ingreso de las familias. Estas disparidades no solo dificultan la construcción de una sociedad más integrada, sino que además afectan los resultados académicos que obtienen los estudiantes (...). (Programa Estado de la Nación en Desarrollo Humano Sostenible, 2008, p.61)

Titulares como el del periódico La Nación del viernes 5 de febrero del año 2010 debería ser suficientemente convincentes respecto de las tareas que es necesario emprender para evitar que los adolescentes sean contratados por las mafias como -de forma quizá exagerada y sin un sustento en datos estadísticos robustos- advirtió, en ese momento, el editorial de La Nación. No obstante, resulta obvio que hay que mejorar las condiciones de vida familiares; la gestión educativa, volviéndola pertinente social y culturalmente, y la provisión de empleos dignos para la ciudadanía.

\section{Conclusiones}

Las conclusiones y recomendaciones que se ofrecen en este apartado condensarán la visión de los distintos actores de la comunidad educativa de las instituciones de enseñanza secundaria visitados.

Las condiciones de pobreza y desempleo son las que se presentan como un obstáculo para la continuidad de los jóvenes en el colegio, independiente de los programas de equidad. Por ello, a pesar de que las familias consultadas ven favorablemente los programas de equidad, estos para algunas de familias resultan insuficientes para sostener a los estudiantes y las estudiantes en las instituciones educativas, independientemente de que haya buen rendimiento académico.

Por otro lado, un factor también determinante de la exclusión se relaciona con la pertinencia educativa, lo cual afecta la motivación. Las ofertas educativas de secundaria no favorecen los intereses específicos del estudiantado, el acceso a estas ofertas se da por proximidad geográfica y no por interés: en la percepción de los jóvenes entrevistados prevalece una mala opinión de lo educativo. El encuentro con las prácticas docentes verticalistas y adultocéntricas, aunado a la poca capacidad de algunos docentes para explicar y mediar la materia que imparten, hace que los jóvenes pierdan interés por el estudio y se afecte su rendimiento académico. 
Por otra parte, mientras lo educativo no está bien considerado ni en general algunos docentes, un motivo fuerte para permanecer en las instituciones son los vínculos con el grupo de pares y amigos. Este gancho es más fuerte que el deseo de aprender, lo que hace cuestionar dos situaciones: en primer lugar, la de pertinencia de la dinámica educativa actual y en segundo lugar, la de las necesidades afectivas y de identidad insatisfecha, que se satisfacen en la vida social que proveen las instituciones.

La ruta crítica hacia el abandono escolar es un proceso complejo, que va vulnerando el interés de los estudiantes y las estudiantes. Abandonar el colegio es una decisión tomada después de pensarlo mucho. Una vez que han tomado tal decisión y sin el apoyo oportuno y coordinado de la familia y la institución, es difícil disuadirles.

Los estudiantes que abandonan el colegio, por lo general, ya lo han hecho en ocasiones anteriores. Si no son los problemas acaecidos con la baja en el rendimiento académico, ante los cual no tiene sentido hacer que la familia siga gastando los pocos recursos con que cuenta, son los problemas de pertinencia educativa, o el interés por aportar recursos a la familia por medio del trabajo. De todos modos, el abandono de los estudios es un fenómeno complejo.

Sin embargo, y aunque están claramente identificados los indicadores de abandono escolar, por parte de las autoridades institucionales, pues saben bien quiénes van a retirarse o abandonar, hay ausencia de mecanismos de prevención y de seguimiento institucional. A pesar de que existe un proceso para atender los casos de abandono escolar, no parece existir una comunicación fluida y coordinada a nivel institucional, para dar seguimiento al joven que se retira de la institución.

En particular y con base las declaraciones dadas por los estudiantes en ese estudio, y por las estadísticas institucionales, es el nivel de sétimo en el que se da mayoritariamente el abandono escolar. Esto, debido a la disminución del apoyo familiar, el incremento del gasto en educación por parte de la familia, el aumento en la cantidad de materias, el número de profesores aunado a los procesos propios del desarrollo psicosocial del joven, entre otros.

Los docentes, por su parte, no son bien percibidos por los estudiantes. En general opinan que no hay empatía con los docentes, y que estos no tienen conocimiento de su realidad comunal y familiar.

El tipo de pedagogía implementada en el aula se identifica como un factor que les desmotiva, pues no logra despertar su interés ni su creatividad. Las orientadoras están recargadas de labores administrativas diferentes a su perfil profesional y sus funciones.

\section{Recomendaciones}

Un conjunto de recomendaciones generales que facilitarían la atención de la exclusión educativa de forma integral y tratando de abordar la multiplicidad de factores que inciden fueron señaladas por los diversos actores educativos: directores, docentes, padres y madres de familia y por supuesto los estudiantes y las estudiantes de secundaria. 
URL: http://www.una.ac.cr/educare

CORREO: educare@una.cr

Lo recientes cambios que se han dado en la evaluación de los aprendizajes son medidas positivas para enfrentar los procesos de exclusión educativa. Hace falta, sin embargo, que la evaluación también pueda valorar aspectos cualitativos que mejoren la apreciación de los aportes del estudiantado.

Ello, junto con la posibilidad de hacer menos vertical la práctica docente y más participativa la mediación, mejoraría el desempeño académico. En relación con los estudiantes y las estudiantes que están al borde del abandono o ya lo han hecho, hay que mejorar la atención psicosocial e implementar un protocolo de atención y seguimiento interinstitucional eficaz.

Por otra parte, es necesario mejorar la selección de los docentes buscando aquellos que puedan trabajar con jóvenes y les ayuden, entre otras cosas, a perfilar sus proyectos de vida con base en la institucionalidad educativa. Esta, por su parte, debería ser lo suficientemente pertinente e interesante para que sea un factor de atracción.

El fortalecimiento de los factores de retención es una acción fundamental que no hay que dejar de lado. Son tres los factores identificados en este estudio que deben favorecerse: a) la motivación del estudiante, entendida como la capacidad del estudiante de mantenerse en la institución a pesar de las dificultades familiares, (b) el apoyo familiar, específicamente a la dinámica familiar con un mejoramiento de las ayudas de tipo socioeconómico que ya se brindan, y c) acciones, a nivel institucional, para la escogencia de profesores, la atención de los sétimos, la adecuación de horario, las actividades recreativas, y la revisión y mejoramiento del currículo (ya se han hecho avances importantes en ese sentido).

\section{Referencias}

Barrantes, T. (1999). Algunas causas de la deserción en los estudiantes que ingresan a 7mo. año en el Instituto Julio Acosta García (Tesis maestría inédita). San José, Universidad de Costa Rica.

Benito, L. (2005). La exclusión escolar desde la percepción de los (as) niños (as) y adolescentes (Tesis de licenciatura inédita). San José, Universidad de Costa Rica.

Castel, R (2004). Encuadre de la exclusión. En S. Karsz (Coomp.). (2004) La exclusión: Bordeando sus fronteras. Definiciones y matices (pp. 55-86). Barcelona, España. Gedisa.

Cortés, R. (1956). La deserción escolar: Un problema fundamental de la democracia costarricense. San José, Costa Rica: Imprenta Tormo.

Fajardo, A. (1968). Rendimiento académico, retención y deserción escolar, desde 1963 y estudio de algunas características de los alumnos de IV y V de 1967 del Liceo Mauro Fernández A. (Tesis de licenciatura inédita). San José, Universidad de Costa Rica.

Flick, U. (2004). Introducción a la investigación cualitativa (Trad. T. del Amo). Madrid: Morata. 
Geldstein, R. N., Loayza, N., Fukazawa, C., Kaztman, R. y Feijoó, M. (2006). Nuevas dinámicas familiares y su impacto en la escolarización de los adolescentes. Debates 4. SITEAL. Recuperado de http://www.siteal.iipe-oei.org/sites/default/files/siteal_libro_debate_4.pdf

Hernández, D. y Barrantes, H. (2002). Deserción escolar en sétimo año: Factores que inciden en la deserción escolar de estudiantes de sétimo año. (Informe de Departamento de Investigación Educativa). San José, Costa Rica: Ministerio de Educación Pública. División de Planeamiento y Desarrollo Educativo.

Irola, A. (2002). Análisis cualitativo de los profesores que provocan deserción escolar en estudiantes de sétimo año del Colegio Técnico de Pacayas (Tesis de maestría inédita). San José: Universidad de Costa Rica.

Jacinto, C. (2009). Consideraciones sobre estrategias de inclusión con igualdad en la escuela secundaria. Debate 7. SITEAL. Recuperado de http://www.ite.educacion.es/formacion/ materiales/126/cd/unidad_7/material_m7/sabermas3.pdf

Jiménez, D., López, I., Saborío, R. y Solano, Y. (1997). Análisis de los factores que causan la deserción escolar en I y ll Ciclo de la Enseñanza General Básica en el circuito 01 de la Dirección Regional de Enseñanza de Puntarenas (Tesis de licenciatura inédita). San José, Universidad de Costa Rica.

Jiménez, R., Robles, E., y Arce, G. (2009). Papel de la educación en el desarrollo económico de Costa Rica. Educación y crecimiento económico en Costa Rica. En L. Mesalles y Céspedes, O (Eds.) (2009). Obstáculos al crecimiento económico de Costa Rica (pp. 181-295). San José, Costa Rica: Academia de Centroamérica.

Loría, J. F., Rodríguez, G., Ureña, J. M. y Ureña, J. B. (1998). El rendimiento académico y la deserción de los estudiantes en el proceso de transición de sexto a sétimo año de la Educación General Básica, en el circuito escolar 06 de la Dirección Regional de Enseñanza de San Ramón en el período 1996-1998 (Tesis de licenciatura inédita). San José, Universidad de Costa Rica.

Ministerio de Educación Pública. Departamento de Análisis Estadístico (2009). Bases de datos de rendimiento académico año 2009. San José de Costa Rica. Autor.

Ministerio de Planificación Nacional y Política Económica (MIDEPLAN). (2007). Plan nacional de desarrollo "Jorge Manuel Dengo Obregón" 2006-2010. San José, Costa Rica: Autor.

Naciones Unidas (s. f.). Podemos erradicar la pobreza 2015. Objetivos de desarrollo del milenio. Recuperado de http://www.un.org/spanish/millenniumgoals

Pérez, R. (1999). De la escuela al colegio: Una guía para fortalecer el proceso de la articulación entre sexto y sétimo de la Educación General Básica (EGB). San José, Costa Rica: MEP-PROMECE.

Picaroni, B. (2009). La evaluación en las aulas de primaria: Usos formativos, calificaciones y comunicación con los padres. Grupo de Análisis para el Desarrollo Recuperado de http:// www.preal.org 
URL: http://www.una.ac.cr/educare

CORREO: educare@una.cr

Programa de promoción de la reforma educativa en América Latina y el Caribe (PREAL). (marzo, 2003). Deserción escolar. Un problema urgente que hay que abordar. Formas y reformas de la educación. Serie Políticas, 5(14), 1-4. Recuperado de http://hdrnet.org/78/1/ Desercion_Escolar.pdf

Programa Estado de la Nación en Desarrollo Humano Sostenible. (2008). Estado de la Educación 2 (2 $2^{\mathrm{a}} \mathrm{Ed}$.). San José, Costa Rica: Consejo Nacional de Rectores.

Real Acdemia Española. (2001). Diccionario de la lengua española (22a ed.). Recuperado de http://lema.rae.es/drae/

Rojas, M. (1998). La inducción al cambio para disminuir la deserción en los estudiantes que ingresan a sétimo año en el colegio Mario Quirós Sasso (Tesis de licenciatura inédita). San José: Universidad de Costa Rica.

Sistema de Información de Tendencias Educativa en América Latina (SITEAL). (2007). La construcción del bienestar de las familias en América Latina: Oportunidad u obstáculo frente a las metas educativas (Cap. 1). En SITEAL (Ed.), Informe sobre tendencias sociales y educativas en América Latina (pp. 29-51). Francia: UNESCO, Argentina: IIPE-UNESCO Sede Regional Buenos Aires, Madrid: OEl. Recuperado de http://www.siteal.iipe-oei.org/ informe/226/informe-2007

Sistema de Información de Tendencias Educativa en América Latina (SITEAL) (2008). Los adolescentes y sus condiciones materiales de vida (Cap. 1). En SITEAL (Ed.), La escuela y los adolescentes. Informe sobre tendencias sociales y educativas en América Latina (pp. 33-60). Francia: UNESCO, Argentina: IIPE-UNESCO Sede Regional Buenos Aires, Madrid: OEl. Recuperado de http://www.oei.es/pdfs/siteal2008.pdf

Sistema de Información de Tendencias Educativa en América Latina (SITEAL). (s. f.). Consulta de base de datos. Recuperado de http://www.siteal.iipe-oei.org/base_de_datos/consulta\#

\section{Cómo citar este artículo en APA:}

Jiménez, W. y Gaete, M. (2013). Estudio de la exclusión educativa y abandono en la enseñanza secundaria en algunas instituciones públicas de Costa Rica. Revista Electrónica Educare, 17(1), 105-128. Recuperado de http://www.revistas.una.ac.cr/index. php/EDUCARE/issue/current

Nota: Para citar este artículo en otros sistemas puede consultar el hipervínculo "Como citar el artículo" en la barra derecha de nuestro sitio web:

http://www.revistas.una.ac.cr/index.php/EDUCARE/index 